\title{
High-dose vitamin-C induced prolonged factitious hyperglycemia in a peritoneal dialysis patient: a case report
}

\author{
Olivier Lachance ${ }^{1 *} \mathbb{0}$, François Goyer ${ }^{2}$, Neill K. J. Adhikari ${ }^{3}$, Marie-Hélène Masse ${ }^{4}$, Jean-François Bilodeau ${ }^{5}$, \\ François Lamontagne ${ }^{4,5}$ and Marc-André Leclair ${ }^{5}$
}

\begin{abstract}
Background: High-dose vitamin C is increasingly used for sepsis and more recently for coronavirus disease 2019 (COVID-19) infections. Proponents argue that the low cost and near perfect safety profile of vitamin C support its early adoption. Yet, adverse events might be underreported and underappreciated.

Case presentation: We report a 73-year-old non-diabetic white man with end-stage renal disease on peritoneal dialysis admitted to the intensive care unit with septic shock that was suspected to be due to peritonitis. The patient was enrolled in LOVIT (Lessening Organ Dysfunction with VITamin C; ClinicalTrials.gov identifier: NCT03680274), a randomized placebo-controlled trial of high-dose intravenous vitamin C. He developed factitious hyperglycemia, as measured with a point-of-care glucometer, that persisted for 6 days after discontinuation of the study drug, confirmed to be vitamin C after unblinding. He also had short-lived iatrogenic coma because of hypoglycemia secondary to insulin administration. These events triggered a protocol amendment.
\end{abstract}

Conclusions: Although factitious hyperglycemia has been reported before using certain glucometers in patients treated with high-dose vitamin C, the persistence of this phenomenon for 6 days after the discontinuation of the therapy is a distinguishing feature. This case highlights the importance of monitoring glucose with a core laboratory assay for up to a week in specific populations, such as patients on peritoneal dialysis.

Keywords: Ascorbic acid, Vitamin C, Intensive care, Septic shock, Sepsis, Peritoneal dialysis

\section{Background}

High-dose vitamin $\mathrm{C}$ therapy for sepsis is being actively debated in the critical care community. A single-center before-after study suggested that high-dose intravenous vitamin $\mathrm{C}$ combined with thiamine and hydrocortisone dramatically decreases mortality, organ failure, and vasopressor requirements in septic shock [1], which was contradicted by a recent multicenter open-label randomized clinical trial [2]. Another trial found that

\footnotetext{
*Correspondence: olivier.lachance.2@umontreal.ca

1 Department of Critical Care Medicine, Université de Montréal, 5400 Boul Gouin Ouest, Montreal, OC H4J 1C5, Canada
}

Full list of author information is available at the end of the article high-dose intravenous vitamin $\mathrm{C}$ reduced 28-day mortality in patients with sepsis and acute respiratory distress syndrome [3].

Skeptics have criticized early reports of efficacy, while enthusiasts argue that the high burden of sepsis and low cost and remarkable safety of vitamin $C$ justify early adoption. Yet, vitamin $C$ therapy has already been associated with factitious hyperglycemia and harmful iatrogenic hypoglycemia $[4,5]$, causing death in at least one report [6]. LOVIT (Lessening Organ Dysfunction with VITamin C) is a multicenter randomized controlled trial (ClinicalTrials.gov identifier: NCT03680274) examining the effects of intravenous vitamin C $(50 \mathrm{mg} / \mathrm{kg}$ every 6 hours for 96 hours) in septic patients in the intensive original author(s) and the source, provide a link to the Creative Commons licence, and indicate if changes were made. The images or other third party material in this article are included in the article's Creative Commons licence, unless indicated otherwise in a credit line to the material. If material is not included in the article's Creative Commons licence and your intended use is not permitted by statutory regulation or exceeds the permitted use, you will need to obtain permission directly from the copyright holder. To view a copy of this licence, visit http://creativecommons.org/licenses/by/4.0/. The Creative Commons Public Domain Dedication waiver (http://creativeco mmons.org/publicdomain/zero/1.0/) applies to the data made available in this article, unless otherwise stated in a credit line to the data. 
care unit (ICU). A patient in this trial had factitious hyperglycemia leading to insulin administration and hypoglycemic coma, which triggered a protocol amendment, highlighting the hazards of such therapy.

\section{Case presentation}

A 73-year-old non-diabetic (HbA1c 5.7\%) white man with end-stage renal disease on peritoneal dialysis was admitted with diarrhea, fever, and hypotension. At home, the patient was on automated nocturnal peritoneal dialysis $(6 \times 2 \mathrm{~L} \times 2.5 \%$ over 8 hours $)$ with $2 \mathrm{~L}$ of icodextrin as a daytime dwell. Physical examination was unremarkable except for abdominal distension. Sepsis due to peritonitis was suspected, and he received intravenous fluids, vasopressors, and broad-spectrum antibiotics. Blood cultures grew Salmonella spp. The patient continued to receive peritoneal dialysis, with three daytime exchanges of $2.5 \%$ or $4.25 \%$ dextrose and one night exchange of icodextrin. The patient had some urine output at baseline but became anuric following hospital admission.

The patient consented to participation in the LOVIT trial 18 hours after admission to the ICU. Before the first dose of study drug, he received subcutaneous insulin due to sepsis-associated hyperglycemia. Capillary blood glucose was monitored with the Accu-Chek Inform II glucometer (F. Hoffmann-La Roche Ltd.). On hospital day 5 , he received intravenous insulin for worsening hyperglycemia, after which he became unconscious. A blood sample sent to the core laboratory and tested using a hexokinase assay showed severe hypoglycemia $(1.7 \mathrm{mmol} / \mathrm{L}$; normal $>4 \mathrm{mmol} / \mathrm{L})$. Insulin was discontinued, 50\% dextrose was administered, and his cognitive status normalized.

After unblinding, research staff confirmed that he had been receiving vitamin $\mathrm{C}$. $\mathrm{He}$ was discharged to the ward on hospital day 10. An ascorbic acid level of $568 \mu \mathrm{mol} / \mathrm{L}$ (normal range: $30-114 \mu \mathrm{mol} / \mathrm{L}$ ) was measured using a spectrophotometric dinitrophenylhydrazine assay 5 days after discontinuing vitamin $C$ therapy, on hospital day 11, and important differences between blood glucose measured by the core laboratory and point-of-care glucometers persisted until hospital day 12 (Fig. 1). Following this event, the LOVIT trial protocol was amended to mitigate the risk of factitious

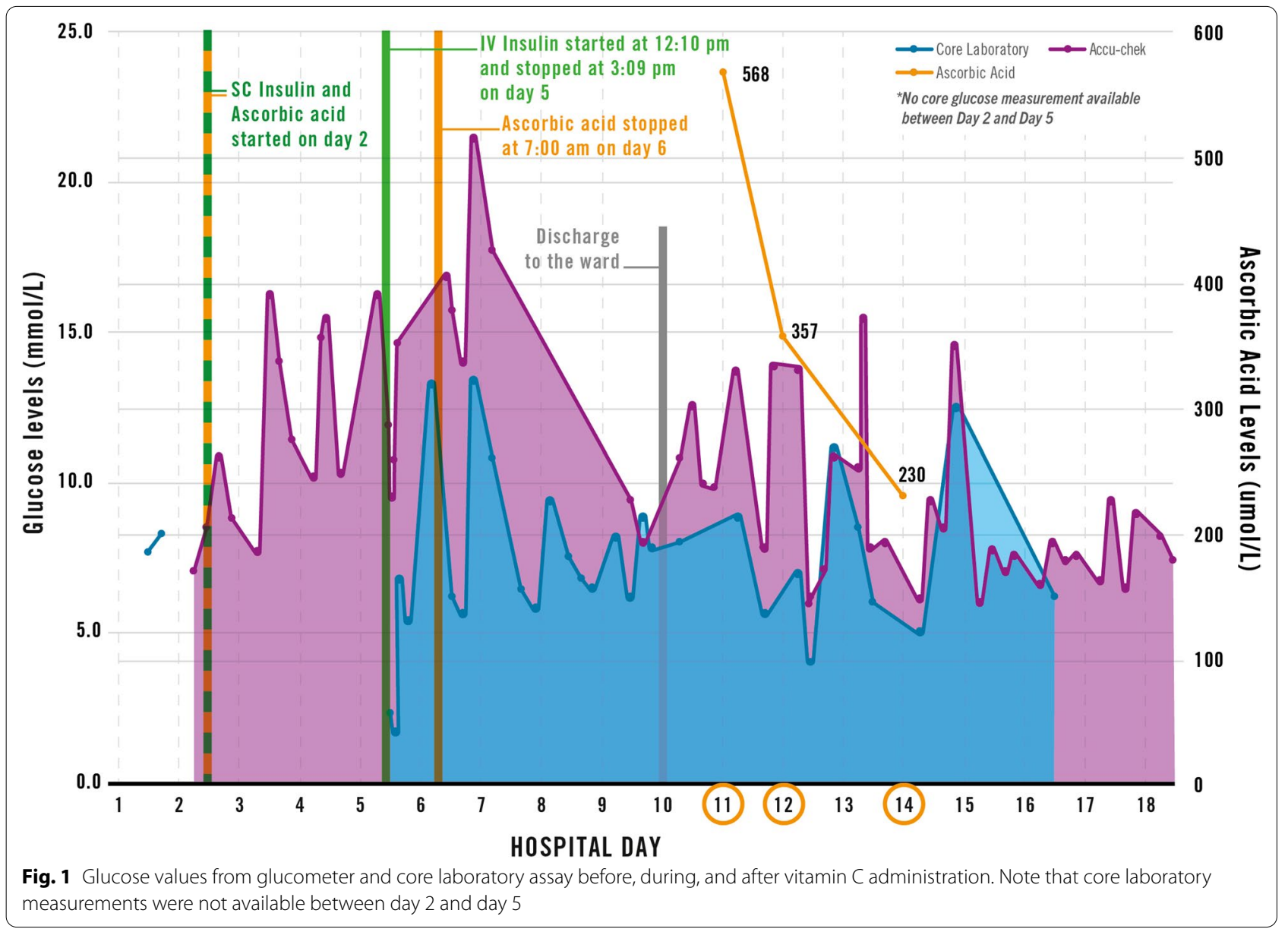


hyperglycemia. The patient was discharged home without any apparent sustained harm.

\section{Discussion}

High-dose vitamin C treatments is increasingly used outside of trials for various conditions such as sepsis and more recently for coronavirus disease 2019 (COVID-19) infections [7]. This case highlights that the adverse effects of high-dose vitamin $C$ treatment may not be fully appreciated and that claims of vitamin C's absolute safety are likely unfounded. Although factitious hyperglycemia on point-of-care glucometers in the presence of elevated plasma ascorbic acid is documented [4, 8], the persistence of factitious hyperglycemia for 6 days after discontinuing vitamin $C$ therapy is a distinguishing feature of this case. An earlier study in patients with burn injury suggested that measuring glucose with a core laboratory method for 24 hours after discontinuation of high-dose vitamin $C$ was a safe approach [4]. The severity and the persistence of this episode suggests that a longer period of monitoring of blood glucose, up to 1 week, might be necessary, perhaps particularly in patients with reduced renal function and those receiving peritoneal dialysis. During this extended period, glucose should be monitored with a core laboratory assay, or other device whose glucose levels are documented to be reliable in the presence of high plasma ascorbic acid concentrations, until no discrepancy with point-of-care glucometers can be demonstrated.

In people with normal kidney function, ascorbic acid is mostly excreted renally [9]. Peritoneal dialysis may have contributed to this patient's course, since pharmacokinetic studies have shown that peritoneal dialysis does not reliably clear plasma oxalic acid, which is a major end product of ascorbic acid oxidation [10]. In addition, icodextrin has been associated with factitious hyperglycemia due to an accumulation of its metabolites, notably maltose [11], although modern glucometers (including the Accu-Chek Inform II) are reportedly impervious to this interference. However, in vitro studies have confirmed significant positive interference on the Accu-Chek Inform II for high plasma concentrations of vitamin $\mathrm{C}$ $(850-1700 \mu \mathrm{mol} / \mathrm{L})$ [8]. The mechanism is related to the electrochemical method of measuring glucose and oxidation of vitamin $C$ at the electrode surface, which creates a current yielding a false signal of hyperglycemia. While some evidence suggests that certain glucometers (for example, StatStrip [Nova Biomedical]) are more reliable than others in the context of high-dose vitamin $\mathrm{C}$ therapy, clinical experience with such devices remains extremely limited [12]. Since glucometers are ubiquitous on medical wards, the cost of acquiring, calibrating, and regularly testing point-of-care devices impervious to ascorbic acid interference will need to be considered as an additional cost of high-dose vitamin $C$ treatment, which may not be justified unless a clear benefit of this treatment can be demonstrated.

This event reinforces the importance of rigorously evaluating novel interventions, even if they are relatively inexpensive and routinely available. The dose of vitamin $\mathrm{C}$ required to improve outcomes in sepsis may exceed that required to normalize plasma levels [13], since scavenging of oxygen radicals and other mechanisms of action may require high intracellular ascorbic acid concentrations. Adverse events are underappreciated and underreported. To mitigate these effects, research should address vitamin C's optimal dosing and pharmacokinetics in critically ill septic patients, including those with renal failure.

\section{Conclusion}

Before the widespread adoption of high-dose vitamin C for the treatment of septic shock, additional randomized trials should assess its safety and potential side effects, which might otherwise be underreported. In certain populations, such as patients undergoing peritoneal dialysis, the risk of persistent factitious hyperglycemia while using point-of-care glucometers is of particular importance. In this population, measurement of blood glucose should be done with core laboratory assay for up to a week after vitamin $\mathrm{C}$ discontinuation.

\section{Abbreviation \\ LOVIT: Lessening Organ Dysfunction with VITamin C.}

\section{Acknowledgements}

We thank Christian Audet for the infographic.

\section{Authors' contributions}

OL contributed to the clinical management of the patient, literature review and manuscript writing. FG contributed to the literature review of the interference between point-of-care glucose monitors and ascorbic acid and contributed to manuscript writing. M-HM contributed to manuscript writing. J-FB contributed with clinical expertise on the case and manuscript revision. NKJA, $\mathrm{M}-\mathrm{AL}$ and $\mathrm{FL}$ contributed to the interpretation of the case, manuscript writing and revisions. All authors read and approved the final manuscript.

\section{Funding}

LOVIT is funded by a grant from the Lotte and John Hecht Memorial Foundation. The funder had no role in the writing of this case report.

\section{Availability of data and materials}

All data generated or analyzed during this study are included in this published article.

\section{Declarations}

\section{Ethics approval and consent to participate}

The LOVIT trial has been approved by the Comité d'éthique de la recherche du Centre intégré universitaire de santé et de services sociaux de l'Estrie - Centre hospitalier universitaire de Sherbrooke (Reference MP-31-2019-2945). The 
patient provided verbal and written consent for his participation in the LOVIT trial.

\section{Consent for publication}

Written informed consent was obtained from the patient for publication of this case report and any accompanying images. A copy of the written consent is available for review by the Editor-in-Chief of this journal.

\section{Competing interests}

Drs. Adhikari and Lamontagne are co-principal investigators of the LOVIT trial.

\section{Author details}

'Department of Critical Care Medicine, Université de Montréal, 5400 Boul Gouin Ouest, Montreal, QC H4J 1C5, Canada. 'Department of Medical Biochemistry, Université de Sherbrooke, 3001 12e Avenue N, Sherbrooke, QC J1H $5 \mathrm{H} 3$, Canada. ${ }^{3}$ Department of Critical Care Medicine, Sunnybrook Health Sciences Centre and Interdepartmental Division of Critical Care Medicine, University of Toronto, 2075 Bayview Ave, Toronto, ON M4N 3M5, Canada. ${ }^{4}$ Centre de recherche du Centre Hospitalier Universitaire de Sherbrooke, 3001 12e Avenue $\mathrm{N}$, Sherbrooke, QC J1H 5H3, Canada. ${ }^{5}$ Department of Medicine, Université de Sherbrooke, 3001 12e Avenue N, Sherbrooke, QC J1H 5H3, Canada.

Received: 7 August 2020 Accepted: 16 April 2021

Published online: 21 May 2021

\section{References}

1. Marik PE, et al. Hydrocortisone, vitamin C, and thiamine for the treatment of severe sepsis and septic shock: a retrospective before-after study. Chest. 2017:151(6):1229-38.

2. Fujii T, et al. Effect of vitamin C, hydrocortisone, and thiamine vs hydrocortisone alone on time alive and free of vasopressor support among patients with septic shock: the VITAMINS randomized clinical trial. JAMA. 2020;323:423.

3. Fowler AA 3rd, et al. Effect of vitamin C infusion on organ failure and biomarkers of inflammation and vascular injury in patients with sepsis and severe acute respiratory failure: the CITRIS-ALI randomized clinical trial. JAMA. 2019;322(13):1261-70.

4. Kahn SA, Lentz CW. Fictitious hyperglycemia: point-of-care glucose measurement is inaccurate during high-dose vitamin $C$ infusion for burn shock resuscitation. J Burn Care Res. 2015;36(2):e67-71.

5. Kim SK, et al. Spurious elevation of glucose concentration during administration of high dose of ascorbic acid in a patient with type 2 diabetes on hemodialysis. Yonsei Med J. 2013;54(5):1289-92.

6. MAUDE Adverse Event Report: ROCHE DIAGNOSTICS ACCU-CHEK ${ }^{\circledR}$ INFORM II TEST STRIPS BLOOD GLUCOSE MONITORING TEST STRIPS. 2017. https://www.accessdata.fda.gov/scripts/cdrh/cfdocs/cfMAUDE/detail. cfm?mdrfoi_id=6767309\&pc=LFR.

7. Peng, Z. Vitamin C infusion for the treatment of severe 2019-nCoV Infected Pneumonia. 2020. NCT04264533. https://clinicaltrials.gov/ct2/ show $/$ NCT04264533?id $=$ NCT04264533\&draw $=2 \&$ rank $=1 \&$ load $=$ cart.

8. Cho J, et al. Influence of vitamin C and maltose on the accuracy of three models of glucose meters. Ann Lab Med. 2016;36(3):271-4.

9. Morgan $\mathrm{SH}$, et al. Oxalate metabolism in end-stage renal disease: the effect of ascorbic acid and pyridoxine. Nephrol Dial Transplant. 1988;3(1):28-32.

10. Mydlik M, Derzsiova K. Renal replacement therapy and secondary hyperoxalemia in chronic renal failure. Kidney Int Suppl. 2001;78:S304-7.

11. Riley SG, et al. Spurious hyperglycaemia and icodextrin in peritoneal dialysis fluid. BMJ. 2003;327(7415):608-9.

12. Ceriotti F, et al. Comparative performance assessment of point-of-care testing devices for measuring glucose and ketones at the patient bedside. J Diabetes Sci Technol. 2015;9(2):268-77.

13. Oudemans-van Straaten HM, Spoelstra-de Man AM, de Waard MC. Vitamin C revisited. Crit Care. 2014;18(4):460.

\section{Publisher's Note}

Springer Nature remains neutral with regard to jurisdictional claims in published maps and institutional affiliations.

Ready to submit your research? Choose BMC and benefit from:

- fast, convenient online submission

- thorough peer review by experienced researchers in your field

- rapid publication on acceptance

- support for research data, including large and complex data types

- gold Open Access which fosters wider collaboration and increased citations

- maximum visibility for your research: over $100 \mathrm{M}$ website views per year

At $\mathrm{BMC}$, research is always in progress.

Learn more biomedcentral.com/submissions 